ISSN: 2386-3919 - e-ISSN: 2386-3927

DOI: http://dx.doi.org/10.14201/et201634193117

\title{
LA GEOMETRÍA A TRAVÉS DEL ARTE EN EDUCACIÓN INFANTIL
}

\section{Geometry through Art in preschool education}

\author{
Álvaro ANTÓN SANCHO* y Manuela GÓmEZ ALONSO** \\ * Profesor de Matemáticas. Escuela Universitaria de Magisterio "Fray Luis de León". \\ Universidad de Valladolid. Correo-e: alvaro.anton@eumfrayluis.com \\ * Maestra de Educación Infantil. CEIP "Jesús del Monte». Hazas de Cesto, Cantabria. \\ Correo-e: manuela.gomez.alonso@eumfrayluis.com
}

Recibido: 30-10-2014; Aceptado: 18-10-2015; Publicado: 30-05-2016

BIBLID [2386-3919 (2016) 34, 1; 93-117]

Ref. Bibl. ÁLVARO ANTÓN SANCHO y MANUELA GÓMEZ ALONSO. La geometría a través del arte en Educación Infantil. Enseñanza \& Teaching, 34, 1-2016, 93-117.

RESUMEN: En este trabajo comprobamos que la expresión artística como recurso didáctico, concretamente la obra de un pintor del entorno próximo al alumnado, permite un aprendizaje significativo y motivador de los contenidos de geometría en la etapa infantil, adquirido además de modo globalizado junto a otras áreas de conocimiento. Para ello, hemos analizado el contenido del currículo de geometría de Educación Infantil, hemos descrito cómo ocurre el aprendizaje infantil de la geometría y aportado unas líneas metodológicas generales para trabajarla. Finalmente, hemos realizado una intervención educativa en el área de geometría sobre un grupo de alumnos de tercer curso de Educación Infantil utilizando la obra pictórica de Julio de Pablo como recurso. Los objetivos principales de la propuesta son presentar de modo lúdico y significativo a través de la obra de nuestro autor los diversos contenidos geométricos (topológicos, proyectivos y métricos), favorecer la observación y exploración del entorno con perspectiva geométrica y el desarrollo de la creatividad, la memoria, la reflexión y el juicio crítico. Hemos evaluado inicialmente las habilidades geométricas, artísticas y de capacidad reflexiva e interpretativa, y, tras realizar la intervención, hemos vuelto a evaluar para comparar los datos. El núcleo de este trabajo consiste en describir la intervención, extraer resultados y 
analizarlos. De ellos colegimos que el recurso a la expresión artística, concretamente a la obra pictórica de un autor del entorno local, permite presentar de modo globalizado, significativo y motivador la totalidad de los contenidos de geometría de Educación Infantil con excelente aprovechamiento por parte del alumnado y de una manera flexible y adaptable a sus necesidades e intereses. Por eso estimamos que una propuesta como la que hacemos es fácilmente adaptable a otros cursos y grupos de Educación Infantil y aprovechable en sus líneas generales por los docentes.

Palabras clave: intervención educativa; Educación Infantil; geometría; arte; aprendizaje significativo.

SUMMARY: In this paper we found that artistic expression as a teaching resource, namely the work of a painter next to students setting, allows meaningful and motivating learning of the contents of geometry in the infant stage, which also is acquired in a globalized way along to other areas of knowledge. To do this, we analyze the content of the curriculum of geometry in the infant stage, describe how children's learning of geometry occurs and provided some general methodological lines to work it. Finally, we conduct an educational intervention in the area of geometry over a group of third year students from kindergarten using the paintings of Julio de Pablo as a resource. The main objectives of the proposal are presented in a fun and meaningful way through our author's work contained the various geometric (topological aspects, projective and metric), encourage observation and exploration of the environment with geometric perspective and the development of creativity, memory, thinking and judgment. We initially evaluated geometric, artistic and reflective skills and acting ability, and following the intervention, we have re-evaluated to compare the data. The core of this paper is to describe the intervention, extract and analyze results. Of these we gather that the use of artistic expression, namely the paintings of a nearby student author, can present globalized, meaningful and motivating way the entire contents of geometry kindergarten with excellent use by students and a flexible and adaptable to their needs and interests so. Therefore we estimate that a proposal like that do is readily adaptable to other courses and groups of child Education and usable in broad outline by teachers.

Key words: educational intervention; preschool; geometry; art; meaningful learning.

\section{INTRODUCCIÓN}

La condición significativa y globalizada del aprendizaje en la Educación Infantil exige contextualizarlo en un ambiente adecuado. Por ello, es necesario crear situaciones de aula que contengan los contenidos curriculares que se quiere enseñar y resulten propicias a la manipulación y experimentación por el alumnado, les resulten motivadoras y se adapten a los ritmos de pensamiento del niño.

Contextualizar el aprendizaje en algún aspecto de su marco sociocultural es una idea clásica y fructífera (Cubero y Luque, 2001). Esta dinámica permite que 
los alumnos vivan experiencias ricas en contenidos y en las que se sientan afectivamente implicados, lo cual aumenta su motivación y facilita un aprendizaje significativo. En el caso de los primeros conceptos geométricos, entendemos que la expresión artística supone una contextualización privilegiada. Las matemáticas y el arte están estrechamente vinculadas (Edo, 2006), sobre todo a través de elementos geométricos (formas, longitudes, simetrías, mosaicos, etc.) y proporciones numéricas. Giménez (2009) explica que esta vinculación ha sido continua a lo largo de la historia. Además, resulta cercano, atractivo y motivador, permite crear situaciones muy diversas y tratar aspectos curriculares muy diversos de forma integrada.

\section{LA GEOMETRÍA EN LA EDUCACIÓN INFANTIL}

\subsection{Las matemáticas en el currículo de Educación Infantil}

La Ley Orgánica 2/2006 de 3 de mayo de Educación (LOE) enfatiza la importancia de la lógica-matemática en la Educación Infantil. En efecto, uno de los objetivos planteados para la etapa de Infantil (el objetivo g) plantea la necesidad de iniciar al alumnado en las habilidades lógico-matemáticas en conexión con otras como la lectoescritura, el movimiento, el gesto y el ritmo. Esta visión es asumida por la LOMCE.

El Real Decreto 1630/2006, por el que se establecen las enseñanzas mínimas del segundo ciclo de Educación Infantil, enmarca el desarrollo de estas habilidades dentro del área correspondiente a Conocimiento del entorno, en particular en el bloque dedicado a conocimiento físico. La ley pide enfocar la enseñanza infantil de las matemáticas (en particular de la geometría) desde una doble perspectiva: por una parte, globalizado e interdisciplinar y, por otra, manipulativo y vinculado al entorno cercano (Edo y Ribeiro, 2007).

\subsection{Observación, exploración y experimentación en la construcción del pensamiento geométrico}

Piaget (1976) explica cómo el desarrollo psicológico en el niño ocurre según un proceso de reorganización de estructuras previas que son puestas en cuestión al contacto con la realidad y los problemas que esta le plantea. De ahí que Ausubel complete esta visión manifestando que el aprendizaje en el niño es prioritariamente significativo, es decir, vinculado a la percepción y a la experiencia (Beltrán, 1998; Edo y Revelles, 2004). Por esa razón, la adquisición de estructuras mentales en el niño exige observación, exploración de la realidad y experimentación (Palacios, Marchesi y Coll, 2001).

En la edad infantil, el aprendizaje es fruto de lo que los discentes observan, exploran y experimentan inmersos en la sociedad (Cubero y Luque, 2001). Hay una diferencia real entre lo que los niños pueden aprender por sí solos (desarrollo real) y lo que pueden aprender aprovechando el entorno social en donde están 
inmersos y sus estructuras (desarrollo potencial). Esta distancia es la denominada zona de desarrollo próximo.

La escuela constructivista señala, como hemos visto, la observación, la exploración y la experimentación como pilares del aprendizaje en la Educación Infantil. Alsina (2006) concreta y sistematiza este enfoque identificando algunas necesidades del niño para la adquisición del pensamiento geométrico. Entre estas necesidades: observar su entorno, vivenciar situaciones de movimiento, manipular y actuar sobre los objetos, simular situaciones reales sobre las que intervenir, jugar, verbalizar lo que hacen y descubren para favorecer la comprensión, aprender en grupo para aprovechar la dimensión social del aprendizaje. Veremos que la expresión artística permite aglutinar todos estos puntos y favorecer, de esta manera, una correcta adquisición de los primeros conceptos de la geometría (Crooks y Alibali, 2014).

Este enfoque exige tratar los contenidos geométricos en la edad infantil de modo interdisciplinar. El aprendizaje en la etapa de Educación Infantil debe ser globalizado, ya que no se pueden separar los aspectos geométricos de los objetos y situaciones del resto de los contenidos (Berdonneau, 2008). Ello nos obliga a buscar y sacar a la luz los contenidos matemáticos de desarrollos como el artístico que, si bien admiten, como hemos explicado, esta dimensión matemática, a primera vista pudiera parecer que esta no es la principal.

Es necesario hacer un apunte en torno a la manera en que tiene lugar la observación en la edad infantil. No es una observación completa, sino que está limitada y condicionada por las características propias de su momento psicológico (Palacios et al., 2001):

- Sincretismo (no pueden realizar una observación analítica completa).

- Contracción (habitualmente se centran en un aspecto de su interés, desestimando todas las demás dimensiones de la realidad).

- Egocentrismo (que brota de la implicación afectiva con lo observado).

Por esa razón el papel del adulto es fundamental. Es necesario ayudar al niño a sistematizar su observación (Alsina, 2006). Esta ayuda ha de ir dirigida en una doble dirección:

- Generalizar la observación, ayudándoles a establecer comparaciones y semejanzas o pidiendo a los niños que se expresen acerca de lo observado.

- Analizar la observación, profundizando así en el contenido de lo observado para ayudarles a extraer de allí conceptos nuevos como formas, medidas o posiciones.

En cuanto a la exploración, hay que decir que brota del interés y la curiosidad natural del niño hacia la realidad circundante. Por eso es necesario potenciar en la intervención educativa este elemento de motivación hacia el descubrimiento de la realidad. A su vez, la exploración ayuda al alumnado a ser protagonista de 
la adquisición de nociones geométricas clave vinculadas con la realidad que le rodea: relaciones entre objetos, formas, comparaciones, tamaños, clasificaciones, ordenaciones, posiciones, etc. Ello debe ser complementado con actividades de experimentación que les permitan plantear hipótesis sobre las formas y los cuerpos y corroborarlas o desestimarlas. De esta manera profundizarán en el conocimiento de estos mismos conceptos geométricos.

\subsection{Evolución del pensamiento geométrico en Educación Infantil}

El pensamiento geométrico en el niño de Educación Infantil se construye de forma progresiva, como corresponde a las características generales de su pensamiento. Además, los conceptos geométricos son elaborados por el niño fundamentalmente a partir de la percepción de sí mismo y de lo que le es próximo (Crooks y Alibali, 2014). Por esa razón, la manera de trabajar la geometría en la edad infantil debe ser principalmente intuitiva y exploratoria (Canals, 1997). El aprendizaje de la geometría se elabora principalmente en dos sentidos: la asimilación de los elementos topológicos, proyectivos y métricos del espacio y la adquisición de las formas geométricas.

En cuanto a la representación geométrica del espacio, hay que decir que el niño evoluciona desde una localización egocéntrica hasta una localización objetiva cada vez más amplia a través de la percepción y la exploración (es el llamado espacio perceptivo que el niño construye durante los periodos sensomotriz y preoperatorio).

La percepción espacial se lleva a cabo de modo sensorial y afectivo-emocional. Además de aprender a orientar y posicionar, el niño proyecta sus gustos y sus emociones en el objeto de su observación, fruto del egocentrismo que gobierna su vivencia. Debemos aprovechar este factor para motivar al niño en su aprendizaje, antes de ayudarle a superarlo para extraer nociones geométricas generales (Sullivan, Clarke, Clarke, Farrell y Gerrard, 2013).

Al organizar el espacio, el niño trabaja dos dimensiones fundamentales de su pensamiento geométrico: la organización espacial y la estructuración espacial.

La orientación espacial consiste en mantener constante la localización del propio cuerpo en relación con la posición del resto de objetos próximos. Consiste, por tanto, en posicionar los objetos en función de la propia posición. Entran en juego, por tanto, las relaciones topológicas, que han de trabajarse a partir del propio cuerpo. En este sentido, obtenemos los tres tipos de relaciones consagradas por Piaget (Holloway, 1969): orientación (izquierda-derecha, delante-detrás, etc.), situación (dentro-fuera, encima-debajo, etc.) y distancia (cerca-lejos, etc.). Pero, además, es importante señalar que todos estos conceptos son adquiridos por el niño paralelamente al conocimiento y dominio corporal.

Por su parte, la estructuración espacial se refiere a la capacidad para situar o posicionar objetos entre sí. Tiene que ver con las relaciones proyectivas, que 
permiten relacionar objetos entre sí en función de una perspectiva dada, y las relaciones métricas, que permiten medir distancias u otras magnitudes.

En lo que se refiere al reconocimiento de formas geométricas, los niños comienzan por clasificar los objetos familiares, desde su propio cuerpo, según ciertas formas geométricas básicas. Estas formas incipientes carecerán de precisión, que se irá adquiriendo paulatinamente. Por ejemplo, se tenderá a confundir todo cuadrilátero con un cuadrado o toda forma redondeada con el círculo (Canals, 1997). El objetivo es que el niño vaya adquiriendo paulatinamente, a través del contacto con los objetos cercanos, una abstracción de estas formas por idealización.

\subsection{Lineas metodológicas}

Vamos a presentar ahora lo que entendemos que deben ser los principios metodológicos básicos que han de orientar el trabajo en el aula con el área de geometría (Wessels, 2014).

1. Intervenir en geometría de modo globalizado con otras materias (Edo y Revelles, 2004). De este modo, la geometría dejará de ser un corpus inamovible para convertirse en un elemento de conocimiento vinculado con la realidad, lo cual favorece la memorización, redunda en la motivación y ayuda a construir un aprendizaje significativo.

2. Partir del propio cuerpo y los propios movimientos. Observar los primeros conceptos a partir del propio cuerpo ayuda a proyectar esos conceptos al espacio circundante general e identificarlos en los objetos que el niño visualiza.

3. Atender siempre a los elementos de la realidad (Cubero y Luque, 2001). El contacto con la realidad debe ser tomado como elemento constante motivador del trabajo de la geometría. Facilita que el niño se interese por el aprendizaje e integre su dimensión afectiva y otorga al niño puntos de anclaje con sus esquemas de conocimientos anteriores, de modo que le permite elaborar conocimiento nuevo lleno de significado (Canals, 1997).

4. Aprovechar todo momento para enseñar geometría (Smole, 2000). Esto favorece que el niño descubra la geometría en los objetos de la naturaleza, en las acciones de la vida cotidiana, en los edificios que visita o en el mobiliario urbano. Una intervención utilizando el arte como recurso facilita especialmente este punto.

5. Priorizar la exploración y la experimentación. Habitualmente se señala como un elemento metodológico indispensable en toda didáctica de la matemática en Infantil priorizar el aprendizaje de procedimientos sobre el de conceptos (Fernández, 2006; Chamorro, 2005). El aprendizaje de procedimientos, que en nuestro caso se llevará a cabo mediante actividades de exploración y experimentación, pretende que el niño llegue a ser capaz de: observar formas y propiedades, descubrir relaciones, posiciones, 
medidas y relaciones comparativas (movimientos, simetrías), así como explorar el espacio y los movimientos.

6. Construir modelos geométricos. Se busca del niño que sea capaz de manipular material de diverso tipo con una intención geométrica (Beltrán Llera, 1998). Este material puede ser de uso corriente o de desecho (este será el caso de nuestra intervención, porque entendemos que aporta un mayor grado de significatividad al aprendizaje), material específicamente geométrico (como el geoplano, el tangram o los policubos) o incluso programas informáticos que permitan visualizar construcciones geométricas.

7. Expresión verbal. Es necesario que todo el aprendizaje que el niño ha ido adquiriendo se estructure en verdadero conocimiento. Mediante actividades adicionales basadas en la expresión verbal, el niño ordena lo aprendido, lo interioriza y vincula su conocimiento con los elementos geométricos, que irá adquiriendo paulatinamente. A su vez, la asimilación del lenguaje geométrico ayuda al niño a un correcto desarrollo del lenguaje general (Alegre, 2002).

8. Atender a la dimensión social del aprendizaje (Cubero y Luque, 2001). Principalmente mediante el trabajo grupal, en gran y pequeño grupo, y con la participación de las familias en el proceso de intervención, en busca del modelo integrador en educación que señaló Gútiez (1995).

La consideración de estos principios metodológicos nos conduce a la necesidad de plantear tres tipos de actividades para una adecuada intervención educativa en el área de geometría en Educación Infantil:

- Actividades de motivación. Son las que facilitan al niño observar los contenidos de la geometría a partir del propio cuerpo, de la realidad y de representaciones historiadas de la realidad como puede ser el cuento.

- Actividades de exploración y experimentación. Permitirán la adquisición de los conceptos geométricos mediante la manipulación y construcción de modelos.

- Actividades de síntesis y generalización de lo observado. Mediante la expresión artística y la verbalización conduce a trabajar la adquisición simbólica de nociones geométricas, la interiorización de los conceptos aprendidos y del lenguaje específico.

\section{ARTE y MATEMÁTICAS EN LA EDUCACIÓN INFANTIL}

\subsection{Arte como recurso didáctico para la enseñanza infantil de la geometría}

Como hemos explicado, la expresión artística goza de un extraordinario contenido matemático. Por esa razón, las obras artísticas pueden ser utilizadas como recurso didáctico para la enseñanza de las matemáticas en diversos niveles 
educativos (Fernández y Reyes, 2003). Pero también ocurre que la perspectiva matemática aporta una luz nueva capaz de aumentar la comprensión y el disfrute de la obra artística.

En las artes plásticas, las obras adquieren su entidad dentro de un espacio y se estructuran en torno a una composición ordenada lógicamente. Por esa razón, el vínculo entre lógica, geometría y belleza se revela particularmente importante en la expresión artística (Vallejo López, 2011).

A través del arte se desarrolla la creatividad y se facilita la observación y reconocimiento de las propiedades geométricas de los objetos. Esto ayuda a generalizar y sistematizar un conocimiento geométrico ordenado que, además, será significativo, porque ha sido adquirido en permanente contacto con la realidad. Permite trabajar, además, de forma globalizada porque se está vinculando al conocimiento matemático otros contenidos (artísticos, naturales y físicos, históricos, etc.) y social, porque el arte permite el diseño de actividades diversas que se pueden llevar a cabo con diferentes tipos de agrupamientos.

Trabajar las matemáticas a través de la expresión artística supone contextualizar de modo muy preciso el aprendizaje, lo que favorece la adquisición de nuevos conceptos (Edo, 2008). La contextualización artística favorece la observación, el análisis e interpretación de formas y distribuciones espaciales y la manipulación o creación de obras de arte facilita la exploración y la experimentación con esos mismos conceptos, de modo que facilitamos el desarrollo natural del aprendizaje del niño.

Además, las competencias geométricas que se pueden desarrollar a través de la expresión artística son numerosas, debido a la riqueza en contenido matemático de que goza habitualmente el arte, como hemos explicado. En las obras de arte podemos encontrar aspectos topológicos, proyectivos y métricos que permiten establecer comparaciones (más largo que, más pequeño que), descubrir relaciones (semejanzas y diferencias), aprender nociones básicas de orientación espacial, utilizar cuantificadores (muchos, pocos, algunos) y también descubrir en los objetos representados propiedades de forma que permitan la adquisición de formas geométricas básicas (Vallejo López, 2011). Por esa razón, resulta un recurso privilegiado a partir del que podemos diseñar actividades adecuadas a los diferentes niveles cognitivos de la educación infantil, primaria y secundaria (Wessels, 2014).

Ilustremos todas estas ideas generales a partir de un artista concreto cuya obra servirá de recurso para nuestra intervención educativa: el pintor cántabro Julio de Pablo (1917-2009). Es un pintor de nuestro tiempo y del entorno local y cultural de los niños sobre los que se realizará la intervención. Se trata de un artista plástico que hizo pintura en óleo y sobre cerámica y también dibujo. Dada su cercanía al mar, extrae de él motivos para sus obras, que destacan por representar con sobriedad formas nítidamente distinguidas a través del color. Encontramos discos centrales negros con manchas rojas o amarillas, círculos centrífugos que recuerdan formaciones cósmicas, formaciones estelares y también formas abstractas: astros, ojos, mejillones, peces, caracolas, velas de barcos, elipses (Caffarena, 1973). Todas esas imágenes están sugeridas como formas geométricas bien identificables. Pero 
es interesante también la disposición de esas formas en los cuadros. Se distinguen giros, simetrías, traslaciones y también distancias y profundidades (por tanto, a la riqueza en formas geométricas se une la presencia de elementos topológicos, proyectivos y métricos).

A continuación aportamos una muestra de su obra (Cerezo y De Pablo, 1999) destacando los elementos geométricos que, de modo principal, aparecen en cada caso y que pueden ser usados en el aula de infantil como recurso.

\section{FIGURA 1}

Formas geométricas en Julio de Pablo
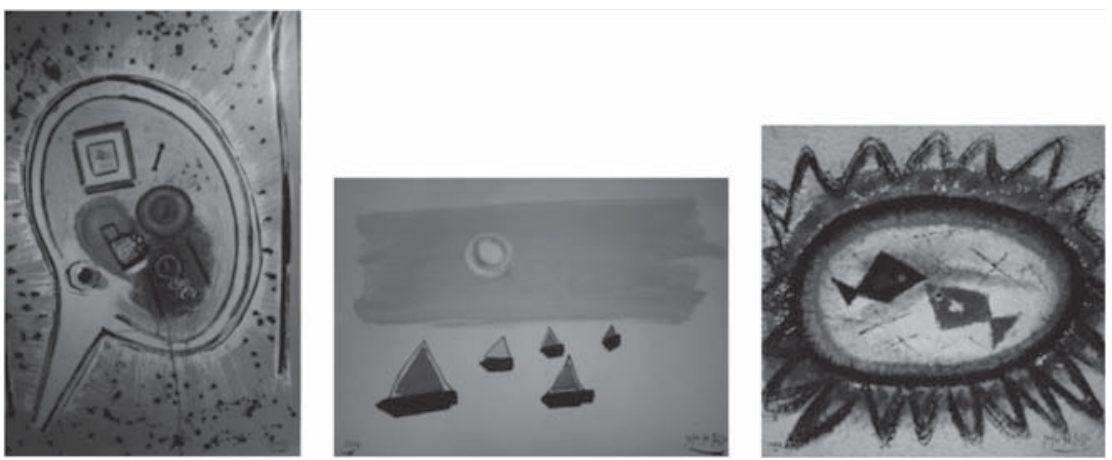

FIGURA 2

Elementos topológicos, proyectivos y métricos en Julio de Pablo

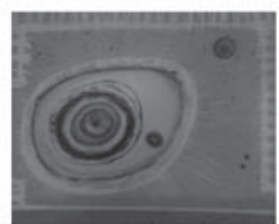

(a) Dentro - fuera

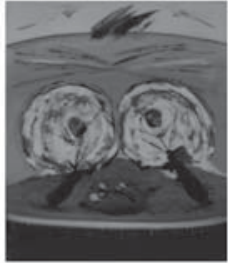

(b) Simetría

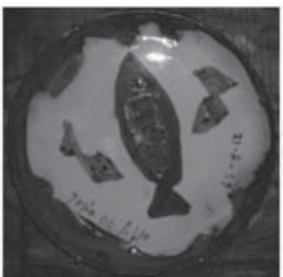

(c) Grande - pequeño

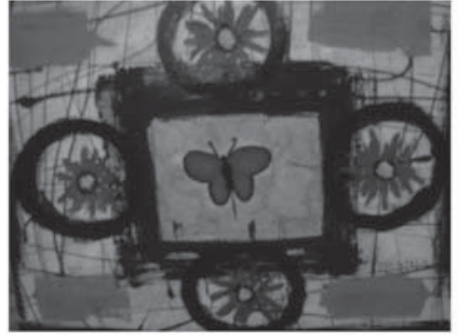

(d) Encima - debajo, derecha - izquierda

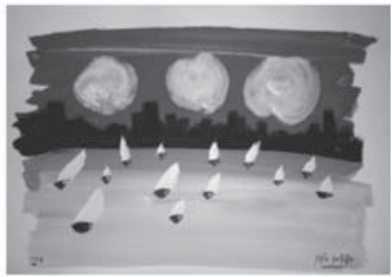

(e) Cerca - lejos, muchos - pocos 
También el cuento ilustrado es un recurso íntimamente ligado a la expresión artística que puede usarse en la didáctica de la geometría infantil. Caballero, Blanco y Blanco (2009) explican que, cuando el cuento acompaña a la ilustración, se aumenta el elemento motivador para el aprendizaje de las matemáticas en general en la edad infantil, se genera una actitud más favorable hacia las matemáticas y se favorece la adquisición de conceptos abstractos (Marín, 1999). La ilustración dentro de los cuentos con contenido matemático permite relacionar el contenido geométrico con situaciones de la realidad, lo que engancha bien con la apelación a la vida real como línea metodológica de motivación. Ayuda, además, a adquirir lenguaje geométrico a través de la expresión estética y fomenta la creatividad y la imaginación, porque esos elementos que los niños ven están integrados dentro de una historia.

Nosotros utilizaremos el cuento infantil ilustrado de contenido matemático $E l$ país de las formas geométricas. Se trata de la historia de diferentes pueblos de figuras geométricas que se van conociendo hasta llegar a formar el país de todas las formas. A partir de las ilustraciones, el alumnado podrá diferenciar triángulos, cuadriláteros y círculos y reconocer posiciones, localizaciones, distancias y tamaños.

\subsection{Antecedentes de la investigación}

Hay diversas propuestas destinadas a diferentes etapas educativas sobre aplicación del arte a la enseñanza de la geometría. Por ejemplo, en Fernández Benito (2010) se describe una propuesta didáctica para la enseñanza de la geometría en la ESO a partir de recursos arquitectónicos (concretamente, lóbulos y polilóbulos). De esta manera logra introducir medidas, fórmulas de áreas, trigonometría y resolución de problemas. La característica fundamental de este trabajo es que lo hace de modo globalizado, pues una propuesta tal le permite el trabajo con todas las competencias básicas. Más recientemente, Antón y Barbado (2015) desarrollaron una intervención en Educación Primaria para ilustrar diversos conceptos de la geometría plana a través de una visita a un complejo arquitectónico.

En la investigación en Educación Infantil destacan las experiencias de Canals (1997) y Edo (2008). Canals (1997) hace un estudio de las estrategias metodológicas básicas para la enseñanza de la geometría en la etapa infantil y primaria y propone algunos ejemplos de actividades de aplicación de esas estrategias. Estas líneas metodológicas sintetizan algunas de las ideas fundamentales que nosotros hemos extraído en nuestro estudio teórico sobre la enseñanza y el aprendizaje de la geometría en la etapa infantil: partir de la realidad, atender a la diversidad (aspecto social), priorizar los aspectos manipulativos (observación y exploración), atender al propio cuerpo y a los propios movimientos y procurar una visión globalizada. Además, entiende que toda intervención de aula debería tener tres fases: movimientos, manipulaciones y dibujos. Nosotros no hemos estructurado nuestras sesiones de esta manera porque hemos querido ser fieles a las fases concretas de aprendizaje del niño. Sin embargo, como veremos, estas fases de Canals estarán presentes de diferentes maneras en nuestra intervención. 
Hay, por tanto, gran comunión entre las líneas generales de Canals y la nuestra. Aunque la propuesta de Canals no utiliza específicamente el arte como recurso didáctico, de hecho usa la expresión artística porque el dibujo de itinerarios, ángulos, figuras, etc., es una constante en las actividades descritas.

Edo (2008) es ya un trabajo en que se trata de aplicar el arte a la enseñanza de las matemáticas en Educación Infantil. En este caso, las actividades propuestas están estructuradas en dos fases. La primera es de observación, análisis e interpretación de obras de arte (se centra en tres autores, dos pintores y un escultor: Joan Miró, Paul Klee y David Smith). La segunda es de creación plástica a partir de la obra analizada. De esta manera se pueden trabajar en el aula algunos aspectos matemáticos, fundamentalmente el reconocimiento de formas geométricas, planas y espaciales.

Nosotros asumimos los principios teóricos y la metodología general que plantea Edo. Sin embargo, introduciremos materiales más variados (como material de reciclaje o el uso de las TIC) y añadiremos a la expresión artística recursos didácticos complementarios, como el cuento, que enriquezcan las actividades y le aporten una perspectiva más globalizadora. Introduciremos como recurso fundamental a un artista local, del entorno cotidiano de los niños: Julio de Pablo. De esta manera aportaremos significatividad al aprendizaje e incidiremos en la dimensión social del mismo. Todo ello nos permitirá elaborar una intervención de aula completa, no sólo una colección de actividades, en que trabajar aspectos más variados de la geometría: reconocimiento de formas y elementos topológicos, proyectivos y métricos.

\section{INTERVENCIÓN EDUCATIVA EN EL AULA}

\subsection{Contexto}

La intervención se ha llevado a cabo durante el curso 2013/2014 en el CEIP "Jesús del Monte» en Hazas de Cesto (Cantabria).

La intervención se realizó en un grupo de tercer curso de Educación Infantil (segundo ciclo, con 5 años). El nivel ha sido elegido así porque trabajaremos un amplio espectro de elementos geométricos, algunos de los cuales requieren de haber adquirido un cierto grado de abstracción.

El aula está formada por 15 alumnos, 7 niñas y 8 niños. Es un grupo cohesionado. No hay apreciables problemas de aprendizaje en ninguno de ellos. Más bien, el aprendizaje es regular, de modo que la evolución de los niños es homogénea. Esto provoca que el nivel inicial de conocimientos sea también homogéneo. Asimismo, es un grupo con alto grado de motivación, que muestra signos de receptividad hacia nuevos aprendizajes. 


\subsection{Instrumentos de la investigación}

Hemos llevado a cabo una investigación cualitativa a través de una intervención de aula. Los instrumentos son la observación sistemática y una actividad de collage de figuras geométricas, que sirvió de evaluación inicial y final y que permitió medir el grado de destreza en cuanto a reconocimiento y diferenciación de formas planas, posiciones y cuerpos geométricos.

La intervención se llevó a cabo en tres bloques, estructurados cada uno en actividades de motivación, desarrollo (exploración y experimentación) y síntesis. Los ejes en torno a los que giraron las actividades fueron:

1. Trabajo a partir del cuento El país de las formas geométricas.

2. Análisis de algunas pinturas de Julio de Pablo.

3. Realizar esculturas con material de reciclaje, en colaboración con las familias.

4. Exposición de las obras realizadas.

La evaluación global se ha llevado a cabo a través de la comparativa de las evaluaciones inicial y final, junto con los datos de la observación.

\subsection{Objetivos generales de la intervención}

Con nuestra intervención de aula pretendemos conseguir los siguientes objetivos generales, que serán precisados en objetivos específicos para cada sesión:

1. Utilizar la expresión artística para trabajar los conceptos geométricos (formas, aspectos topológicos, proyectivos y métricos) desde una perspectiva significativa, en relación a la vida cotidiana.

2. Presentar los conceptos geométricos de manera lúdica.

3. Favorecer en los niños, a través del arte, la observación, exploración y experimentación para facilitar la adquisición de las nociones geométricas.

4. Desarrollar la creatividad y la memoria a través de la geometría.

5. Favorecer el análisis y la discriminación de formas geométricas.

6. Motivar el desarrollo de la reflexión y el pensamiento crítico.

7. Abrir la escuela al entorno cultural y artístico, de modo que el aprendizaje de la geometría se haga en un contexto globalizado.

8. Trabajar diferentes agrupamientos, desarrollando así actitudes cívicas que favorezcan la dimensión social del aprendizaje.

9. Introducir las TIC en el contexto educativo general.

\subsection{Materiales y recursos}

En cada sesión especificaremos el material concreto del que haremos uso. Sin embargo, es posible establecer un listado de recursos generales de la propuesta: 
- El propio cuerpo. La primera fuente de relaciones, posiciones y formas es el cuerpo propio y el del compañero.

- Objetos de reciclaje y recogidos de su entorno: botellas de plástico, envases, bolsas, tubos, pelotas, cajas, etc.

- Tecnologías de la Información y la Comunicación (ordenador, reproductor de DVD, cañón y cámara de fotos).

- Material de expresión plástica (témperas, plastilina, ceras, cartulinas, tijeras).

- Recursos espaciales: aula ordinaria, pasillos, recibidor, patio y aula de usos múltiples.

\subsection{Evaluación inicial}

Para llevar a cabo la evaluación inicial del alumnado, llevamos a cabo la actividad titulada collage de figuras geométricas. Se trata de repartir por la alfombra cartulinas con diferentes figuras geométricas dibujadas, de diversos tamaños y colores. Se trata de recortar las figuras y realizar una composición con ellas para, posteriormente, analizar el resultado de su creación. Analizamos qué formas aparecen, los elementos de cada figura (vértices, lados, ángulos), sus características, su tamaño y su posición relativa. Se obtuvieron los siguientes resultados:

TABLA 1

Reconocimiento de formas

\begin{tabular}{|l|c|c|c|c|c|c|}
\hline & \multicolumn{2}{|c|}{ Reconocen la forma } & \multicolumn{2}{c|}{ Número de lados } & \multicolumn{2}{c|}{ Número de vértices } \\
\hline & $\begin{array}{c}N^{\circ} \\
\text { alumnos }\end{array}$ & Porcentaje & $\begin{array}{c}N^{\circ} \\
\text { alumnos }\end{array}$ & Porcentaje & $\begin{array}{c}N^{\circ} \\
\text { alumnos }\end{array}$ & Porcentaje \\
\hline Círculo & 15 & $100 \%$ & - & - & - & - \\
\hline Triángulo & 15 & $100 \%$ & 15 & $100 \%$ & 15 & $100 \%$ \\
\hline Cuadrado & 15 & $100 \%$ & 15 & $100 \%$ & 14 & $93,3 \%$ \\
\hline Rectángulo & 11 & $73,3 \%$ & 15 & $100 \%$ & 14 & $93,3 \%$ \\
\hline Rombo & 12 & $80 \%$ & 15 & $100 \%$ & 13 & $86,6 \%$ \\
\hline
\end{tabular}

Los alumnos reconocen casi todas las figuras geométricas, aunque empiezan a surgir dificultades a la hora de diferenciar entre los cuadriláteros: surgieron dudas sobre si los lados del rombo eran todos iguales, confunden el rectángulo con el cuadrado cuando hay una distinción pequeña entre la medida de los lados y no aciertan a dar una respuesta coherente cuando se les plantea la diferencia entre el cuadrado y el rombo. 
En cuanto al uso del vocabulario geométrico (lados, vértices), todos los alumnos conocen y usan correctamente el término lado pero sólo 3 niños usan correctamente el término vértice. El resto sólo fue capaz de identificar el elemento una vez oída la respuesta de sus compañeros.

En cuanto a la capacidad para diferenciar unas formas de otras:

TABLA 2

Diferenciación de formas

\begin{tabular}{|l|c|c|}
\hline & $N^{\circ}$ alumnos & Porcentaje \\
\hline Círculo - polígono & 15 & $100 \%$ \\
\hline Triángulo - cuadrilátero & 14 & $93,3 \%$ \\
\hline Cuadrado - rectángulo & 10 & $66,7 \%$ \\
\hline Rectángulo - rombo & 8 & $53,3 \%$ \\
\hline Cuadrado - rombo & 0 & $0 \%$ \\
\hline
\end{tabular}

Observamos que, en general, son capaces de distinguir los polígonos por el número de lados, pero tienen problemas a la hora de discriminar los cuadriláteros entre sí por la medida de sus lados o de sus ángulos.

Finalmente, evaluamos la capacidad de distinguir elementos de posición relativa entre figuras geométricas:

TABLA 3

Reconocimiento de posiciones

\begin{tabular}{|l|c|c|}
\hline & $N^{\circ}$ alumnos & Porcentaje \\
\hline Arriba - abajo & 15 & $100 \%$ \\
\hline Dentro - fuera & 15 & $100 \%$ \\
\hline Delante - detrás & 13 & $86,7 \%$ \\
\hline Derecha - izquierda & 8 & $53,3 \%$ \\
\hline
\end{tabular}

Hay algunas cuestiones de posición (en concreto, la distinción entre derecha e izquierda y la dimensión de profundidad) que les crea una mayor dificultad. De los 8 alumnos que supieron distinguir entre derecha e izquierda, sólo 5 (el 33,3\%) supieron identificar esta diferencia en un cuadro. 
En este momento se les mostraron diversos cuerpos geométricos realizados en objetos cotidianos presentes en el aula. Se les pidió que reconocieran el cuerpo y sus elementos notables y que diferenciaran unos cuerpos de otros.

TABLA 4

Cuerpos geométricos

\begin{tabular}{|c|c|c|}
\hline & $N^{\circ}$ alumnos & Porcentaje \\
\hline $\begin{array}{ll}\text { Reconocen } & \text { cuerpos } \\
\text { geométricos } & \text { (esfera, } \\
\text { cilindro, prisma, pirámide) }\end{array}$ & $\begin{array}{c}4 \text { (reconocen la esfera) } \\
2 \text { (esfera y cilindro) }\end{array}$ & $\begin{array}{c}26,7 \% \text { (reconocen la } \\
\text { esfera) }\end{array}$ \\
\hline $\begin{array}{l}\text { Reconocen elementos } \\
\text { notables de los cuerpos } \\
\text { (bases, caras, vértices) }\end{array}$ & 2 & $13,3 \%$ \\
\hline $\begin{array}{l}\text { Reconocen el número de } \\
\text { bases y sus formas }\end{array}$ & 2 & $13,3 \%$ \\
\hline
\end{tabular}

En general, tienen dificultad para distinguir cuerpos geométricos de figuras planas y también para distinguir los cuerpos entre sí. En general, sólo reconocieron la esfera y unos pocos alumnos el cilindro. También les cuesta reconocer los elementos notables de los cuerpos. Existe una confusión generalizada entre caras y lados. Pero tienen una intuición inicial de cuerpo, porque bastantes de ellos los reconocen en su realidad cotidiana.

\subsection{Desarrollo de la intervención}

La intervención se llevó a cabo durante el mes de febrero de 2014. Se realizó en tres bloques. El primero ocupó una sesión, el segundo (programado inicialmente para dos días) ocupó tres y el tercero llevó tres sesiones. 
TABLA 5

Primer bloque

\begin{tabular}{|l|l|}
\hline \multicolumn{1}{|c|}{ TítULO: EL PAÍS DE LAS FORMAS GEOMÉTRICAS } \\
\hline \multirow{6}{*}{ Objetivos } & $\begin{array}{l}\text { - Reconocer formas geométricas planas (círculo, triángulo, cuadrado, } \\
\text { rectángulo, rombo). } \\
\text { - Clasificar y describir objetos del aula por su forma geométrica. } \\
\text { - Relacionar los objetos cotidianos con las formas geométricas. } \\
\text { - Utilizar adecuadamente vocabulario geométrico (lado, vértice, curva, } \\
\text { etc.). } \\
\text { - Desarrollar la motricidad fina. } \\
\text { - Aprender a trabajar en equipo. }\end{array}$ \\
\hline \multirow{6}{*}{ Contenidos } & $\begin{array}{l}\text { - Figuras geométricas planas. } \\
\text { - Agrupaciones y clasificaciones de elementos por forma, tamaño, } \\
\text { número de lados y vértices. }\end{array}$ \\
\hline - Vocabulario geométrico.
\end{tabular}


TABLA 6

Segundo bloque

\begin{tabular}{|c|c|}
\hline \multicolumn{2}{|r|}{ TÍTULO: SOMOS ESCULTORES } \\
\hline Objetivos & $\begin{array}{l}\text { - Reconocer y analizar las características de los objetos con los que se } \\
\text { van a realizar las esculturas. } \\
\text { - Discriminar elementos topológicos (de orientación, situación y } \\
\text { distancia). } \\
\text { - Discriminar elementos proyectivos (posiciones). } \\
\text { - Discriminar elementos métricos (longitudes, superficies, volúmenes). } \\
\text { - Expresar verbalmente las acciones realizadas y las propiedades } \\
\text { geométricas descubiertas en el entorno. } \\
\text { - Crear sus propias esculturas utilizando figuras geométricas. } \\
\text { - Representar a través de la expresión plástica los elementos geométricos } \\
\text { de posición y forma y cambios de posición y forma. } \\
\text { - Potenciar la sensibilidad estética y vincularla al conocimiento } \\
\text { geométrico. } \\
\text { - Valorar el medio ambiente, usando material de desecho. } \\
\text { - Colaborar y participar en actividades grupales. } \\
\text { - Compartir material y respetar el material del otro. } \\
\text { - Canalizar adecuadamente la afectividad en la creación artística. } \\
\text { - Introducir a la familia en la dinámica de aprendizaje del niño. }\end{array}$ \\
\hline Contenidos & $\begin{array}{l}\text { - Agrupaciones y clasificaciones de elementos según la forma, el tamaño, } \\
\text { el número de lados, caras o vértices. } \\
\text { - Conceptos espaciales de orientación, situación y distancia. } \\
\text { - Posición y cambio de posición. } \\
\text { - Comparación de medidas de longitud y superficie. } \\
\text { - Vocabulario geométrico sobre formas espaciales (cara, base, vértice). } \\
\text { - Arte: la escultura. }\end{array}$ \\
\hline $\begin{array}{l}\text { Criterios de } \\
\text { evaluación }\end{array}$ & $\begin{array}{l}\text { - Es capaz de realizar una escultura a partir de elementos geométricos. } \\
\text { - Reconoce aspectos topológicos espaciales, relaciones de proximidad y } \\
\text { compara medidas. } \\
\text { - Utiliza vocabulario geométrico básico. } \\
\text { - Muestra sensibilidad estética. } \\
\text { - Desarrolla actitudes de cuidado del medio ambiente. } \\
\text { - Muestra sensibilidad estética y la vincula al orden geométrico. } \\
\text { - Colabora en actividades grupales y valora las producciones propias y } \\
\text { las de los demás. }\end{array}$ \\
\hline
\end{tabular}




\begin{tabular}{|c|c|c|}
\hline \multicolumn{3}{|c|}{ TÍTULO: SOMOS ESCULTORES } \\
\hline \multirow{3}{*}{ Actividades } & Motivación & $\begin{array}{l}\text { - Se disponen en el centro del aula diversos } \\
\text { objetos que los niños han traído de casa y del } \\
\text { entorno con la colaboración de la familia. }\end{array}$ \\
\hline & $\begin{array}{l}\text { Desarrollo: } \\
\text { exploración y } \\
\text { experimentación }\end{array}$ & $\begin{array}{l}\text { - Clasificar los objetos según su forma (plana o } \\
\text { espacial). } \\
\text { - Nombrar las características de cada objeto } \\
\text { (número de lados, número de vértices, número } \\
\text { y forma de las caras). } \\
\text { - Se hacen cuatro grupos. Cada niño elige dos } \\
\text { objetos para realizar una escultura. } \\
\text { - Experimentar con los objetos: cambio de } \\
\text { posición entre ellos, si encajan o no (medida), } \\
\text { si se apoya uno en otro (forma), etc. } \\
\text { - Por grupos, se realizan las esculturas. }\end{array}$ \\
\hline & Síntesis & $\begin{array}{l}\text { - Palpar con los ojos cerrados la forma de la } \\
\text { escultura. } \\
\text { - Mostrar la escultura al resto de compañeros y } \\
\text { explicar el título, las figuras que la componen, } \\
\text { los objetos que se han utilizado y cómo se han } \\
\text { dispuesto. }\end{array}$ \\
\hline
\end{tabular}

TABLA 7

Tercer bloque

Título: PINTAMOS CON JULIO DE PABLO

- Interpretar obras de arte conocidas a partir de la aplicación de los conceptos geométricos.

- Recordar y representar elementos geométricos que el alumno ha visto con anterioridad en un cuadro.

- Reconocer y representar elementos topológicos (de orientación, situación y distancia).

- Reconocer y representar elementos proyectivos (posiciones).

Objetivos

- Reconocer y representar elementos métricos (longitudes, superficies, volúmenes).

- Utilizar vocabulario geométrico básico.

- Desarrollar la observación, la memoria y la expresión plástica.

- Motivar la reflexión y el pensamiento crítico.

- Buscar información en Internet.

- Respetar y valorar las producciones propias y las de los compañeros. 


\begin{tabular}{|c|c|c|}
\hline \multicolumn{3}{|c|}{ TÍTUlo: PINTAMOS CON JULIO DE PABLO } \\
\hline Contenidos & \multicolumn{2}{|c|}{$\begin{array}{l}\text { - Biografía y obra pictórica de Julio de Pablo. } \\
\text { - Figuras geométricas planas y espaciales. } \\
\text { - Reconocimiento y representación de elementos topológicos, } \\
\text { proyectivos y métricos. } \\
\text { - Uso de Tic (ordenador e Internet). }\end{array}$} \\
\hline Criterios de evaluación & \multicolumn{2}{|c|}{$\begin{array}{l}\text { - Reconoce y representa gráficamente formas geométricas } \\
\text { observadas en diferentes cuadros. } \\
\text { - Reconoce y reproduce orientaciones, situaciones, } \\
\text { localizaciones, posiciones y distancias. } \\
\text { - Busca información en Internet. } \\
\text { - Reconoce aspectos de la vida y la obra de Julio de Pablo. }\end{array}$} \\
\hline \multirow[t]{3}{*}{ Actividades } & Motivación & $\begin{array}{l}\text { - Buscar información sobre Julio de Pablo } \\
\text { en Internet. Buscar información sobre } \\
\text { su vida y su obra. } \\
\text { - Observar las pinturas y hablar sobre los } \\
\text { elementos geométricos que se perciben. } \\
\text { - Indagar sobre los sentimientos e ideas } \\
\text { que quiere transmitir el artista con esas } \\
\text { formas y colores. } \\
\text { - Elegir cada uno la que más llame la } \\
\text { atención y explicar la razón. } \\
\text { - Fomentar un diálogo en que los } \\
\text { alumnos expliquen lo que ven en la } \\
\text { obra. Diálogo dirigido (¿qué título le } \\
\text { pondrías?, ¿a qué te recuerda el cuadro?, } \\
\text { ¿cuántos planos ves?, ¿cuál crees que } \\
\text { es el principal y por qué?, ¿qué formas } \\
\text { aparecen y cuál es la que más se repite?, } \\
\text { ¿cómo están colocadas las figuras?). } \\
\text { - Mostrar alguna obra que no pertenezca } \\
\text { a Julio de Pablo. Deben adivinar cuáles } \\
\text { son estas obras y por qué. }\end{array}$ \\
\hline & $\begin{array}{l}\text { Desarrollo: } \\
\text { exploración y } \\
\text { experimentación }\end{array}$ & $\begin{array}{l}\text { - Después de haber elegido un cuadro, } \\
\text { dibujar evocando el cuadro elegido. } \\
\text { Se pide recordar colores, formas, } \\
\text { posiciones y medidas y representarlas. }\end{array}$ \\
\hline & Síntesis & $\begin{array}{l}\text { - Exponer y explicar las producciones de } \\
\text { cada uno al resto de compañeros. } \\
\text { - Realizar una exposición con todas las } \\
\text { obras. }\end{array}$ \\
\hline
\end{tabular}

La última actividad de la intervención consiste en organizar una exposición con las obras pictóricas que el alumnado ha realizado. Ello obliga a: 
- Preparar un catálogo (por tanto, clasificar las obras según sus elementos geométricos comunes).

- Distribuir las obras espacialmente en el lugar destinado para la exposición.

- Escoger formas de las obras para realizar carteles e invitaciones.

\subsection{Evaluación final y resultados}

Se llevó a cabo una observación directa y sistemática de los alumnos en el desarrollo de las sesiones. Además, al final realizamos las mismas pruebas que se efectuaron en la evaluación inicial. Obtuvimos los siguientes resultados:

TABLA 8

Reconocimiento final de formas

\begin{tabular}{|l|c|c|c|c|c|c|}
\hline & \multicolumn{2}{|c|}{ Reconocen la forma } & \multicolumn{2}{c|}{ Número de lados } & \multicolumn{2}{c|}{ Número de vértices } \\
\hline & $\begin{array}{l}N^{\circ} \\
\text { alumnos }\end{array}$ & Porcentaje & $\begin{array}{c}N^{\circ} \\
\text { alumnos }\end{array}$ & Porcentaje & $\begin{array}{c}N^{\circ} \\
\text { alumnos }\end{array}$ & Porcentaje \\
\hline Círculo & 15 & $100 \%$ & 15 & $100 \%$ & 15 & $100 \%$ \\
\hline Triángulo & 15 & $100 \%$ & 15 & $100 \%$ & 15 & $100 \%$ \\
\hline Cuadrado & 15 & $100 \%$ & 15 & $100 \%$ & 15 & $100 \%$ \\
\hline Rectángulo & 15 & $100 \%$ & 15 & $100 \%$ & 15 & $100 \%$ \\
\hline Rombo & 15 & $100 \%$ & 15 & $100 \%$ & 15 & $100 \%$ \\
\hline
\end{tabular}

Mediante el arte hemos conseguido vencer la dificultad principal que presentaban los alumnos: discernir cuadriláteros según la medida de sus ángulos y lados. Observamos, por tanto, que la observación y expresión artística es eficaz para la adquisición de las competencias en reconocimiento de formas y de medidas. Esto se confirma al visualizar los resultados sobre diferenciación de formas geométricas: 
TABLA 9

Diferenciación final de formas

\begin{tabular}{|l|c|c|}
\hline & $N^{\circ}$ alumnos & Porcentaje \\
\hline Círculo - poligono & 15 & $100 \%$ \\
\hline Triángulo - cuadrilátero & 15 & $100 \%$ \\
\hline Cuadrado - rectángulo & 15 & $100 \%$ \\
\hline Rectángulo - rombo & 14 & $93,3 \%$ \\
\hline Cuadrado - rombo & 14 & $93,3 \%$ \\
\hline
\end{tabular}

Observamos un notable avance también en las competencias referidas a elementos proyectivos del espacio (posición).

TABLA 10

Reconocimiento final de posiciones

\begin{tabular}{|l|c|c|}
\hline & $N^{\circ}$ alumnos & Porcentaje \\
\hline Arriba - abajo & 15 & $100 \%$ \\
\hline Dentro - fuera & 15 & $100 \%$ \\
\hline Delante - detrás & 15 & $100 \%$ \\
\hline Derecha - izquierda & 15 & $100 \%$ \\
\hline
\end{tabular}

Todos los alumnos son ya capaces de discernir entre derecha e izquierda e identificar profundidades.

Finalmente, hemos logrado un avance significativo también en la adquisición de la noción de cuerpo (volumen) y en la identificación de formas planas en la superficie de los cuerpos. 
TABLA 11

Reconocimiento final de cuerpos

\begin{tabular}{|l|c|c|}
\hline & $N^{\circ}$ alumnos & Porcentaje \\
\hline $\begin{array}{l}\text { Reconocen cuerpos } \\
\text { (esfera, cilindro, prisma, } \\
\text { pirámide) }\end{array}$ & 13 & $86,7 \%$ \\
\hline $\begin{array}{l}\text { Reconocen los elementos } \\
\text { notables de los cuerpos } \\
\text { (bases, caras, vértices) }\end{array}$ & 12 & $80 \%$ \\
\hline $\begin{array}{l}\text { Reconocen el número de } \\
\text { bases y sus formas }\end{array}$ & 14 & $93,3 \%$ \\
\hline
\end{tabular}

Además del notable avance en la adquisición de las competencias geométricas lograda con la intervención en un contexto artístico, el hecho de localizar en un ambiente cotidiano la intervención ha conseguido aumentar en los discentes la capacidad para asociar los conceptos de la geometría con su experiencia cotidiana.

TABLA 12

Relación de la geometría con la experiencia

\begin{tabular}{|l|c|c|}
\hline & $N^{\circ}$ alumnos & Porcentaje \\
\hline $\begin{array}{l}\text { Clasifican y describen } \\
\text { objetos del aula por su } \\
\text { forma }\end{array}$ & 14 & $93,3 \%$ \\
\hline $\begin{array}{l}\text { Relacionan objetos } \\
\text { cotidianos con las formas } \\
\text { geométricas }\end{array}$ & 13 & $86,7 \%$ \\
\hline $\begin{array}{l}\text { Interpretan obras de arte } \\
\text { conocidas a partir de } \\
\text { conceptos geométricos }\end{array}$ & 15 & $100 \%$ \\
\hline
\end{tabular}

Relacionan satisfactoriamente los objetos que perciben con los contenidos dados porque son capaces de ver los elementos geométricos en los objetos que perciben. Esto aumenta su motivación para trabajar en cuestiones geométricas y, por tanto, su predisposición para el aprendizaje de la materia. 


\section{CONClusiones}

El hecho de utilizar el arte como hilo conductor de esta intervención educativa para trabajar la geometría ha favorecido el mantenimiento de un alto nivel de motivación durante su aplicación debido al elemento significativo que introduce y a su carácter lúdico. Permite, además, gran flexibilidad para ajustar la experiencia a los objetivos pretendidos y a los intereses de los alumnos en el mismo momento de estar realizándola. Se logran, asimismo, altos grados de significatividad en el aprendizaje. Además, la obra de Julio de Pablo goza de una gran riqueza de elementos geométricos, lo que hace posible integrar, en torno a un mismo recurso didáctico, una amplia colección de contenidos del área de geometría. En particular, hemos podido presentar de forma interrelacionada elementos de forma y aspectos topológicos, proyectivos y métricos. Todo ello conduce a que se pueda alcanzar una buena adquisición de la globalidad de los conocimientos geométricos en poco tiempo.

La experiencia relatada muestra que los contenidos de la geometría y de la expresión plástica se enriquecen mutuamente, de modo que podemos adquirir amplias cuotas de conocimiento en ambos campos interviniendo de manera globalizada.

En particular, de la evaluación final se deduce que se ha conseguido mejorar capacidades como la creatividad y la memoria a través de la utilización del arte como recurso didáctico. Asimismo, el alumnado ha demostrado haber desarrollado la reflexión y el sentido crítico general, como se deduce de los resultados expuestos en la Tabla 12.

Entendemos que nuestra experiencia permite confirmar y ahondar en trabajos previos como el de Edo (2008), pero esta vez extrayendo datos cuantitativos concretos que fundamenten nuestras conclusiones. Hemos conseguido, además, diseñar una colección de actividades en torno al mismo autor, lo que permite relacionar entre sí los contenidos geométricos y no presentarlos, como en ocasiones se hace, a través de actividades ocasionales y fortuitas. Asimismo, a experiencias anteriores como Edo (2008), Antón y Barbado (2015) o Fernández (2010) hemos añadido el uso de diversos recursos multimedia, lo que favorece la motivación y el desarrollo de competencias digitales. De este modo, hemos integrado elementos que ya han propuesto intervenciones como la de Antón y Duque (2015). También cabe destacar que nuestro recurso artístico es conocido por el alumnado. Creemos que este tipo de actividades facilitan un acercamiento al entorno cultural y ayudan a desarrollar en los discentes una diversidad de perspectivas para el análisis de su realidad circundante.

Cabe destacar también la importancia de mantener un esquema de intervención estructurado según el modo de aprender de los niños: motivación, desarrollo (observación, exploración y experimentación) y síntesis (reflexión, análisis). No queremos decir que haya que ganar rigidez en la organización de las actividades. Al contrario. Pero creemos que es necesario adecuar la estructura general de la 
intervención al ritmo de aprendizaje del niño y entendemos que los resultados obtenidos y expuestos en este trabajo avalan esta percepción.

En conclusión, el uso del arte permite intervenir en el área de geometría desde una perspectiva plástica y sociocultural, lo que suscita en los alumnos experiencias de riqueza matemática, pero también social, creativa, visual, afectiva, que les ayudarán a asimilar los contenidos geométricos transidos de sentimiento estético.

\section{REFERENCIAS BIBLIOGRÁFICAS}

Alegre, J. R. (2002). Maestro infantil: desarrollo del pensamiento lógico-matemático. Deslogmat. Descargado el día 25 de octubre de 2014. http://www.juntadeandalucia.es/ averroes/ cepco3/competencias/mates/infantil/razonamiento\%201\%F3gico-matematico.pdf.

Alsina, A. (2006). Cómo desarrollar el pensamiento matemático de 0 a 6 años. Barcelona: Octaedro.

Antón, Á. y Barbado, P. (2015). Geometría en el arte: el prerrománico asturiano. UNO. Revista de Didáctica de las Matemáticas, 68, 67-74.

Antón Sancho, Á. y Duque Domingo, J. V. (2015). Intervención sobre la suma mediante el uso combinado de regletas y TIC’s. En C. Fernández, M. Molina y N. Planas (Eds.). Investigación en Educación Matemática, XIX (p. 533). Alicante: SEIEM.

Beltrán Llera, J. (1998). Procesos, estrategias y técnicas de aprendizaje. Madrid: Síntesis.

Berdonneau, C. (2008). Matemáticas activas (2-6 años). Barcelona: Graó.

Caballero, A.; Blanco, B. y Blanco, J. L. (2009). Matemáticas a través de los cuentos. Aula de Innovación Educativa, 188, 79-95.

Caffarena, A. (1973). Gloria Torner y Julio de Pablo, dos pintores montañeses. Málaga: Dardo.

Canals, M. A. (1997). La geometría en las primeras edades escolares. Suma, 25, 31-44.

Cerezo, A. y De Pablo, J. (1999). Elucidario de Julio de Pablo. Santander: Calima.

Chamorro, M. C. (2005). Didáctica de las matemáticas. Madrid: Pearson Prentice Hall.

Crooks, N. y Alibali, M. W. (2014). Defining and measuring conceptual knowledge in mathematics. Developmental Review, 34 (4), 344-377.

Cubero, R. y Luque, A. (2001). Desarrollo, educación y educación escolar: la teoría sociocultural del desarrollo y del aprendizaje. En C. Coll, J. Palacios y A. Marchesi (Eds.). Desarrollo psicológico y educación, 2. Psicología de la educación escolar (pp. 137-155). Madrid: Alianza.

Edo, M. (2006). Matemática y Arte, un contexto interdisciplinario. En Actas del I Congreso Internacional de Lógico-Matemática en Educación Infantil. Madrid: World Assotiation of Early Childhood Educations.

Edo, M. (2008). Matemáticas y arte en la Educación Infantil. Uno, 47, 37-53.

Edo, M. y Revelles, S. (2004). Situaciones matemáticas potencialmente significativas. En M. Antón y B. Moll (Eds.). Educación Infantil. Orientación y recursos (O-6 años) (pp. 103179). Barcelona: Cisspraxis.

Edo, M. y Ribeiro, C. (2007). A Matemática na Educação Infantil: contextos criativos de aprendizagem. En Actas CIANEI, 2. ${ }^{\circ}$ Congresso Internacional de Aprendizagem na Educação de Infância (pp. 595-606). Porto: Gailivro.

Fernández, I. (2010). Polilóbulos y competencias básicas. Suma, 63, 21-28. 
Fernández, I. y Reyes, E. (2003). Geometría con el hexágono y el octógono. Granada: Proyecto Sur.

Fernández, J. A. (2006). Didáctica de la matemática en la educación infantil. Madrid: Mayéutica.

Giménez, J. (2009). La proporción, arte y matemáticas. Barcelona: Graó.

Gútiez, P. (1995). La educación infantil: modelos de atención a la infancia. Revista Complutense de Educación Infantil, 6 (1), 101-113.

Holloway, G. E. T. (1969). Concepción del espacio en el niño según Piaget. Buenos Aires: Paidós.

Marín, M. (1999). El valor del cuento en la construcción de conceptos matemáticos. Números. Revista de Didáctica de las Matemáticas, 39, 27-38.

Palacios, J.; Marchesi, A. y Coll, C. (2001). Desarrollo psicológico y educación. Madrid: Alianza.

Piaget, J. (1976). El nacimiento de la inteligencia en el niño. Madrid: Aguilar.

Piaget, J. (1979). Seis estudios de psicología. Barcelona: Seix-Barrall.

Smole, K. C. (2000). A matemática na educação infantil. A teoría das inteligencias múltiplas na práctica escolar. Porto Alegre: Artmed.

Sullivan, P.; Clarke, D. J.; Clarke, D. M.; Farrell, M. y Gerrard, J. (2013). Processes and priorities in planning mathematics teaching. Mathematics Educational Research Journal, 25, 457-480.

Vallejo López, F. (2011). Las matemáticas en el arte: su didáctica. Revista Digital Ciencia y Didáctica, 50, 73-83.

Wessels, H. M. (2014). Levels of mathematical creativity in model-eliciting activities. Journal of Mathematical Modeling and Applications, 1 (9), 22-40. 
